\title{
SENSOR GUIDED MICRO ASSEMBLY BY USING LASER-SCANNING TECHNOLOGY
}

Sven Rathmann, Jan Wrege, Kerstin Schöttler, Annika Raatz and Jürgen Hesselbach

Institute of Machine Tools and Production Technology (IWF), Technical University Braunschweig, Langer Kamp 19b, 38106 Braunschweig, Germany

Abstract: To recognize geometric objects of components in assembly processes, in particular of microelectronic or micro system components, nowadays the use of vision systems is preferred. These systems are working very fast but the results depend on ambient conditions, especially of light settings. They deliver 2D object information referring to the image plane of a camera. In most sensor guided assembly systems, additional to vision systems laser displacement sensors are implemented to get information about the third dimension, the components height. In this paper a scanning method is presented which enables object recognition by using a laser positioning sensor and to use the achieved measuring values for a sensor guided assembly process.

Key words: precision assembly; laser scanning; in process.

\section{INTRODUCTION}

The used laser-scanning method is a technology to scan surfaces or bodies by using a laser beam. In most cases the laser is deflected by a mirror unit. Alternatively the laser or rather the object is moved. Three different methods are known in laser-scanning technology: confocal laser-scanning, 3D-laser-scanning technology as well as laser altimetry. The most interesting technologies for scanning micro components are the confocal laser-scanning and the 3D-laser-scanning. With these technologies it is possible to obtain exact models with sub micrometer accuracy of investigated micro components. A disadvantage of the named methods is that it is not possible to scan the object during production process. However, in high precision assembly processes it is necessary to get exact in-process information about 
these micro components, for example their position and geometry and the deviation of geometry. For this purpose vision systems can be used. The disadvantage of them is the dependence of light settings. Especially in case of complex geometrical structures with different surface reflection properties, which often occur in micro systems, wrong geometric properties can be detected. For this reason the laser-scanning technology is used to get the desired information about position and deviation of micro components in an assembly process. In high precision assembly systems laser displacement sensors are often implemented. So it is possible to extend the existing assembly systems with a technology in following called In-Process LaserScanning (IPLS).

\section{CONFIGURATION AND METHODS}

IPLS uses a laser, which is mounted on a high accurate micro assembly robot in z-direction of the robot coordinate system (Fig. 1). The sensor is usually used to measure the height of components. In this case it will be used for the scanning process by moving the robot over the component. During IPLS the heights' information of the laser sensor is related to the robot position in $\mathrm{x}$ - and $\mathrm{y}$-direction. The accuracy of the scanning process is mainly addicted to the diameter of the laser beam. For experiments, a confocal laser displacement sensor (Keyence, LT-9001) was used. It has a laser beam diameter of $2 \mu \mathrm{m}$ with a measurement range in $\mathrm{z}$-direction of $\pm 0.3 \mathrm{~mm}$. Additionally to the measurement of the height via a point, the LT9001 offers a line scan option. The integrated horizontal oscillating system deflects the laser beam $\pm 550 \mu \mathrm{m}$ in $\mathrm{x}$-direction to get height information over the deflected range by an increment size down to $2 \mu \mathrm{m}$.

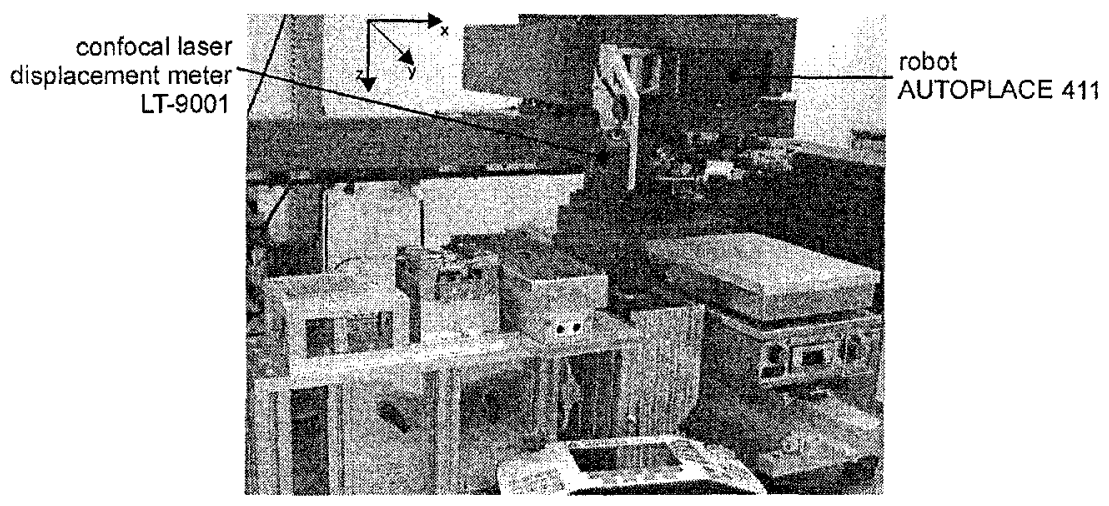

Figure 1. Assembly system with mounted laser displacement sensor (LT-9001) 


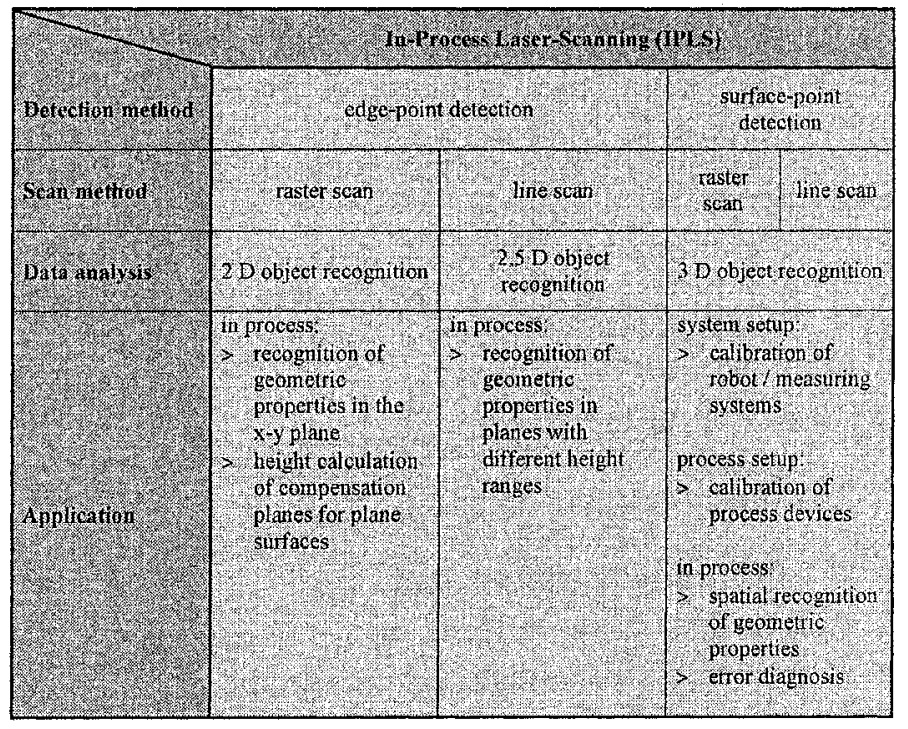

Figure 2. Overview of In-Process Laser-Scanning options

The laser is mounted on a micro assembly robot AUTOPLACE 411 (Sysmelec). The robot offers a repeatability in $\mathrm{x}$-, $\mathrm{y}$ - and z-direction of $1 \mu \mathrm{m}^{1}$. The smallest step size is $0.5 \mu \mathrm{m}$. The laser is connected with the robot control via analog interface. The setup of the high accurate robot and the confocal laser displacement sensor offer a range of scanning options. Fig. 2 shows an overview of those different scanning options.

The IPLS is classified in two detection methods. First the simple edgepoint detection and second the detection of a surface-point of a component. With the edge-point detection and the two scan methods, raster scan and line scan, it is possible to get information about the components' position and their deviation by using a $2 \mathrm{D}$ or $2.5 \mathrm{D}$ data analysis for the object recognition. The raster scan method works with the point laser of the LT9001 and the movement of the robot in $x$ - and $y$-direction. The raster is defined either by a step size in $x$-direction or $y$-direction or both (Fig. 3a). The line scan method uses the line scan option of the LT-9001. The oscillating laser gives information in $\mathrm{z}_{1}$ - and $\mathrm{x}_{1}$-direction while the robot moves only in y-direction (Fig. 3b).

By using the raster scan method an edge-point is detected if the laser height signal is changing significantly during moving over the edge. The data analysis of several edge-points allows the reconstruction of geometric properties of the component in the two dimensions $x_{r}$ and $y_{r}$ related to the robot coordinate system. 

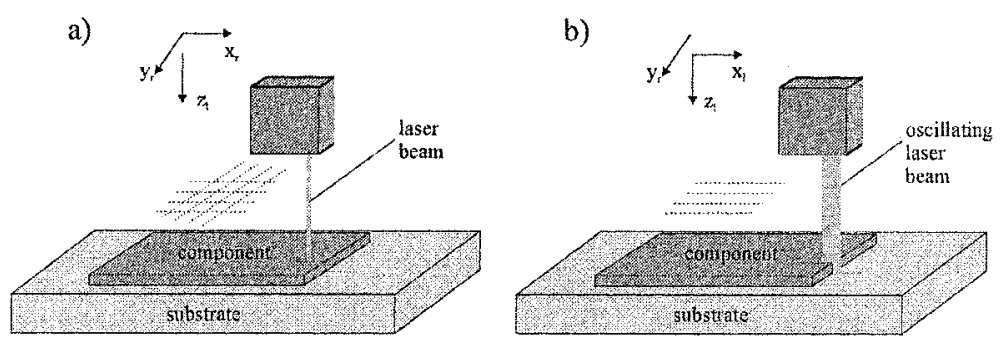

Figure 3. Illustration of the scanning process (a) point scan, (b) line scan

The line scan method allows an exact description of the edge geometry. With this method it is possible to detect edges in spite of disruptions or blurred transition, for instance by evaluating a chosen feature of the edge geometry. This provides an improved stability of the object recognition. In contrast to the raster scan method, the analysis of the line scan information is addicted to a high computation power because of complex mathematical calculations like data projection and coordinate transformation. A reconstruction of the components' geometry can be done using the dimensions $\mathrm{x}_{\mathrm{r}}$ and $\mathrm{y}_{\mathrm{r}}$ additionally with the filtered $\mathrm{z}_{\mathrm{l}}$-dimension (2.5 D).

An enhancement of the edge-point detection is the surface-point detection. This detection works with the coordinates of the robot, the height information of the sensor system and the analysis of this information to get 3D component information. The surface-point detection works also with the raster scan or the line scan method. Using the raster scan method, the robot moves with defined increments in $\mathrm{x}_{\mathrm{r}}$ - and $\mathrm{y}_{\mathrm{r}}$-direction and detects the height information $z_{1}$ of the component with the laser sensor. The 3D object recognition reconstructs the desired information, for example component position, edge geometry and surface planarity, from the measured scatter plot.

In contrast to the raster scan method, the surface-point detection in combination with the line scan method works much faster. The movement of the robot is restricted to $\mathrm{y}_{\mathrm{r}}$-direction. Because of the small scanning range of the oscillated laser sensor $( \pm 550 \mu \mathrm{m})$ this method is only appropriate for surveying of micro components. Using the relation between the $x_{1}$ and $z_{i}$ laser information and the $x_{r}$ and $y_{r}$ robot information, a reconstruction of the $3 \mathrm{D}$ component information related to the robot coordinate system is possible.

\section{APPLICATION}

In Fig. 2 an overview of the possible applications with the different detection and scan methods is shown. 
Edge-point detection with raster scan and line scan is especially used for the detection and recognition of geometric parameters of micro components. With different moving strategies of the robot and analyzing tools recognition, for example of rectangular or circular geometries is possible. In the following chapter an example for the recognition of rectangular geometry is described. Also raster scan can be used for measurements of the height of a component on different positions. The result from this information can be used to adjust the component plane to the working plane. Because of the easy integration into existing micro assembly systems and the simple and fast recognition of object parameters, this detection method is especially qualified for sensor guided assembly.

Surface-point detection can be used for a detection of 3D information of micro components. An advantage of the raster scan method is its possibility to adjust the increment size in $x_{r}-$ and $y_{\mathrm{r}}$-direction in a wide range. This allows a scanning over a larger area, for example to measure the waviness of component surfaces. This scan method can be use for error diagnosis in assembly systems as well as for an enhanced detection of geometric properties especially in plane uses.

The small increment size of the laser line option down to $2 \mu \mathrm{m}$ can be used for a high accurate measurement of micro components. Because of the oscillating principle of the laser optic, the scanning process works without any edge shadowing. For this reason objects with high edge steepness can be detected. An application area can be the calibration of robot axes or implemented devices, like transport devices. Also the setup of new assembly processes or the error diagnosis is possible. Furthermore, the set of $3 \mathrm{D}$ geometric information allows an enhanced object and geometric recognition especially in different planes. Chapter 3.2 describes an exemplary $3 \mathrm{D}$ measurement of a micro component.

\subsection{Edge-Point Detection with Raster Scan}

The prior aim of IPLS is to detect the exact position of component edges in the robot coordinate system. Therefore a routine for the AUTOPLACE 411 was programmed. It comprises the following steps:

1. Move close to the edge

2. Move in increments in direction to the edge

3. Read laser signal at the reached position

4. Break movement, if laser signal changes significantly (edge is reached)

5. Store actual robot position 

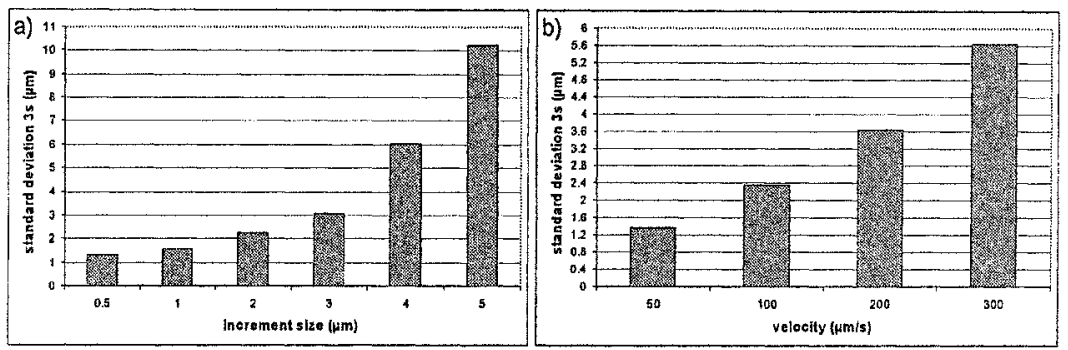

Figure 4. Standard deviation for (a) different increment sizes, (b) different velocities

The reference of the laser to the robot coordinate system was made by a calibration via vision system, which was implemented into the robot's head and was calibrated by manufacture. The vision system has a resolution of 2 $\mu \mathrm{m}$. After scanning different edge-points at the component it is possible to calculate straight lines, intersections, diameter and angels in the assembly plane.

To validate the scanning method a test component, as shown in Fig. 3, was used. This hybrid component consists of a ceramic substrate and a semiconductor chip with a height of merely $100 \mu \mathrm{m}$. The semiconductor chip is smaller than the ceramic substrate. Thus the light contrast between chip and substrate is not adequate to detect edges with a standard vision system.

To get the accuracy of the scanning system, measurements were carried out referring to ISO/TS $14253-2^{2}$. In Fig. 4 a the accuracy with variation of the increment size during the movement to the edge is displayed. Additionally to the laser beam diameter, the accuracy of the scanning process is addicted to the increment size as shown in the figure. This can be traced back to the strict separation between movement and measurement.

The time to detect one point of the edge is addicted on the distance between starting point and edge. It averages 15 seconds per edge-point with an increment size of $2 \mu \mathrm{m}$ and a distance to the edge of about $300 \mu \mathrm{m}$.

A variation to the raster scan method is to move with a constant low speed from the starting point to edge. In Fig. $4 \mathrm{~b}$ the accuracy of the raster scan method with different velocities is shown. With this variation the accuracy could be increased.

The reason for that is a continuous checking of the sensor signal during movement. So the accuracy is not addicted to the increment size but rather to the addiction of the movement's velocity to the runtime of the robot control. The required time to reach the edge-point using continuous movements is 1.5 seconds with a velocity of $200 \mu \mathrm{m} / \mathrm{s}$ and the same distance to the edge as in the raster scan method. 


\subsection{Surface-Point Detection with Line Scan}

To show the capability of the surface-point detection using the line scan method a micro component was scanned. This micro component is a stator of a horizontal micro electric linear actor developed by the IMT, Braunschweig, within the Collaborative Research Center $516^{3}$. The actor is working according to the reluctance principle. It mainly consists of a ceramic stator with six coil-systems meandering horizontally around soft magnetic poles. This stator is fixed to a silicon die which has two v-grooves used as ball tracks. The critical assembly task is to insert the passive pole rows of an associated traveler between the active pole rows of the stator. The horizontal gap between the pole-teeth amounts $3 \mu \mathrm{m}$ over a length of approximately $6 \mathrm{~mm}$. After complete assembly of the actor the gap is adjusted by four slide bars attached to the stator and the traveler. With $40 \mu \mathrm{m}$ depth of insertion a resulting vertical clearance $<2 \mu \mathrm{m}$ occurs.

In Fig. 5 the measurement of one and a half coil-system of the stator is shown. The measurement was done with a laser resolution in $\mathrm{x}_{1}$-direction of $2 \mu \mathrm{m}$ and a robot step size in $\mathrm{y}_{\mathrm{r}}$-direction of $2.5 \mu \mathrm{m}$. An interesting information of those measurements is the edge steepness, which can be resulted with this method up to $92^{\circ}$. Analyzing the scatter plot the edge steepness is in range of the measured values by Seidemann ${ }^{4}$.

In Fig. 6 a scatter plot of a layer horizontal through the scatter plot of Fig. 5 in a height of 30 to $45 \mu \mathrm{m}$ is shown. Analyzing the filtered scatter plot edge recognition is possible.

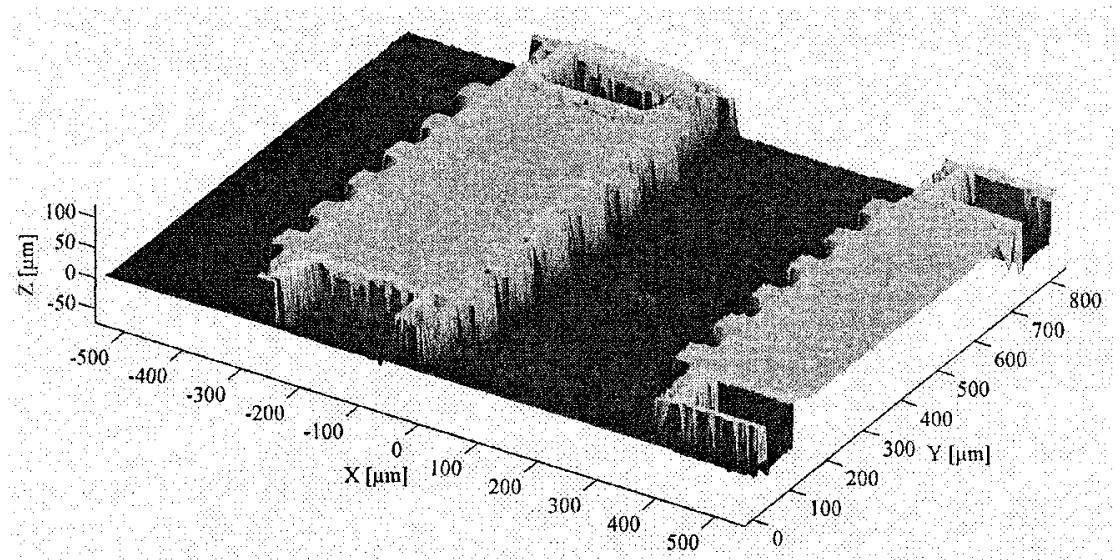

Figure 5. Scatter plot of the micro actuator 


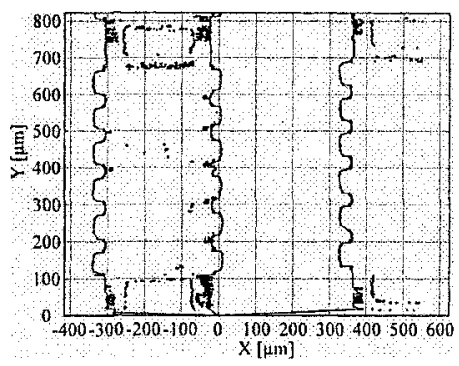

Figure 6. Scatter plot of a horizontal layer of the micro actuator

This information can be used to setup the assembly process, for example to detect starting points for scanning with edge-point detection or to leveling the assembly plane.

\section{CONCLUSION}

In this paper a laser-scanning technology, called In-Process LaserScanning, is presented which can be used in process for measuring micro components. This information can be used for sensor guided assembly. For this method a confocal laser displacement sensor and a high precision robot or tool machine is required. Many micro assembly systems use a laser displacement sensor to get information about the height of components. Therefore the implementation of IPLS is obvious. In this work a laser sensor with a beam diameter of $2 \mu \mathrm{m}$ and a robot with a repeatability of $1 \mu \mathrm{m}$ were used. Different detection and scanning methods are presented and validated with experiments. It could be shown that these scanning methods are adequate for micro assembly.

\section{REFERENCES}

1. J. Hesselbach, G. Pokar, J. Wrege, K. Heuer, Some Aspects on the Assembly of Active Micro Systems, Production Engineering. Research and Development, issue 11, book 1 WGP e.V., Braunschweig, pp. 159-164 (2004).

2. ISO/TS 14253-2, Geometrical Product Specifications (GPS) - Inspection by measurement of workpieces and measuring equipments - Part 2, issue 1999.

3. V. Seidemann, J. Edler, S. Büttgenbach, H.-D. Stölting, Linear Variable Reluctance (VR) Micro Motor with Horizontal Flux Guidance: Concept, Simulation, Fabrication and Test, 12th International Conference on Solid-State Sensors, Actuators and Microsystems, Transducers '03, Boston 2003, pp. 1415-1418.

4. V. Seidemann, Induktive Mikrosysteme: Technologieentwicklung und Anwendung, (Shaker Verlag, Aachen, Germany, 2003). 